\title{
Synthesis and characterization of cardanol-based methacrylate oligomers for three-dimensional printing resins
}

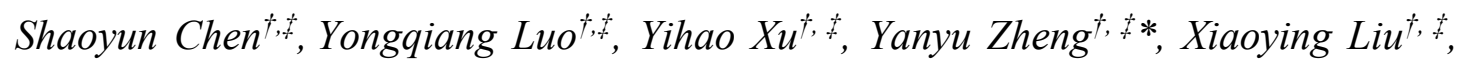
Rui Wang ${ }^{\dagger, *}$, Bo Qu ${ }^{\dagger, *}$, Dongxian Zhuo ${ }^{\dagger, * *}$

${ }^{\dagger}$ College of Chemical Engineering and Materials Science, Quanzhou Normal University, Quanzhou, Fujian, 362000, P. R. China.

${ }^{\ddagger}$ Fujian University Engineering Research Center of Polymer Functional Coating based Graphene, Fujian, 362000, P. R. China.

*Corresponding Authors:

*Email: dxzhuo@quztc.edu.cn

*Email: yy410529@163.com

Supporting Information contains 8 pages including 2 Figures and 2 Tables.

\section{Index}

Figure S1. GPC curevs of MCPPs.

Figure S2. Adhesion of MCPP-3 resin to different substrates

Table S1. The GPC results of different MCPPs

Table S2. Comparison of MCPP resins and other biobased and petroleum-based acrylates for $3 \mathrm{D}$ printing in literature. 


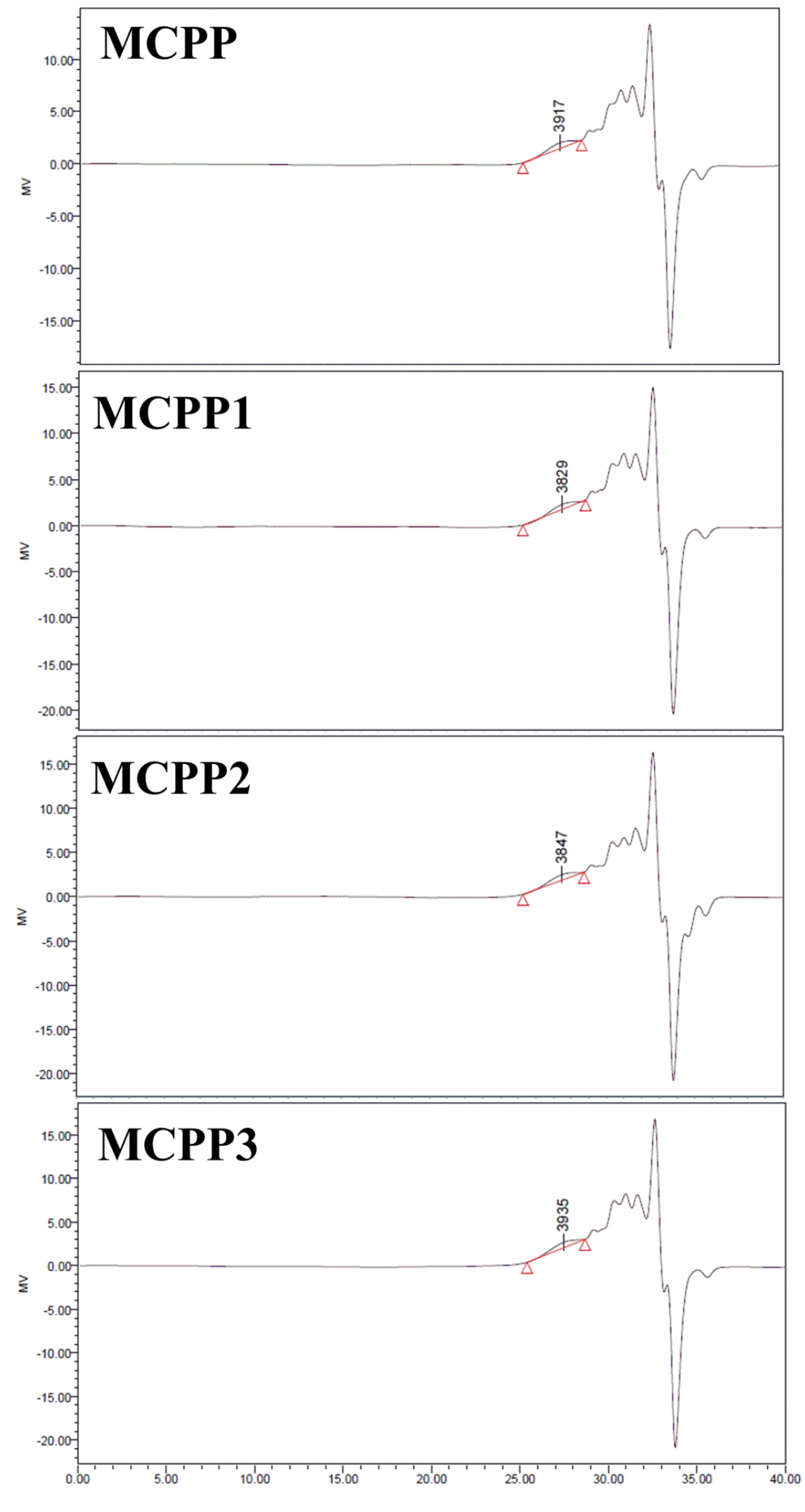

Figure S1. GPC curevs of MCPPs 


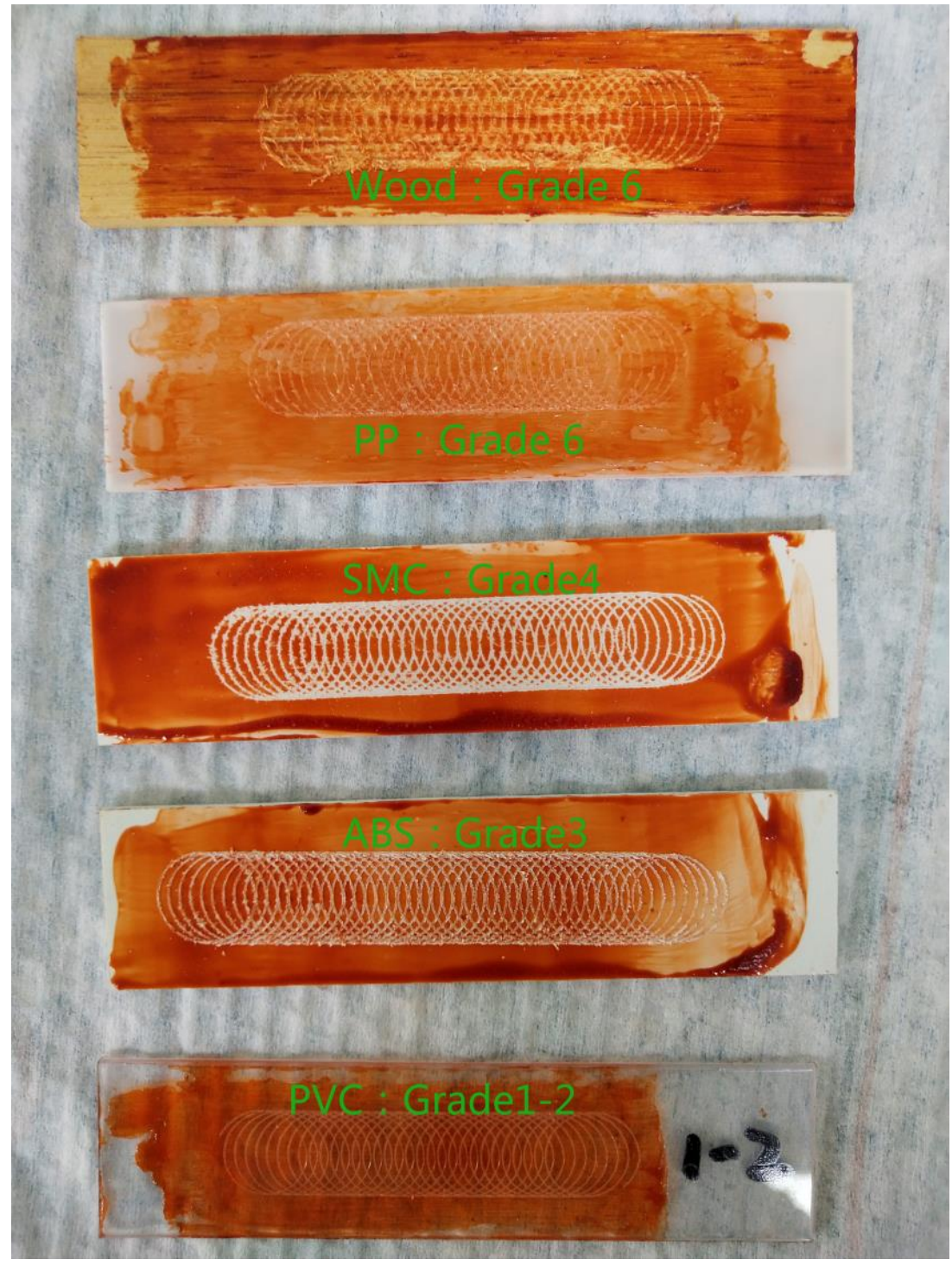

Figure S2. Adhesion of MCPP-3 resin to different substrates 
Table S1. The GPC results of different MCPPs

\begin{tabular}{cccc}
\hline Samples & Mn & Mw & PI \\
\hline MCPP & 3833 & 4162 & 1.08 \\
MCPP1 & 3755 & 4024 & 1.07 \\
MCPP2 & 3814 & 4164 & 1.09 \\
MCPP3 & 3850 & 4200 & 1.09 \\
\hline
\end{tabular}


Table S2. Comparison of mechanical and thermal properties of 3D printed samples based on different photosensitive inks

\begin{tabular}{|c|c|c|c|c|c|}
\hline Samples & $\begin{array}{c}\text { Tensile } \\
\text { strength } \\
\text { (MPa) }\end{array}$ & $\begin{array}{c}\text { Elongation } \\
\text { at break } \\
(\%)\end{array}$ & $\begin{array}{l}\mathbf{T}_{-5 \%} \\
\left({ }^{\circ} \mathbf{C}\right)\end{array}$ & $\begin{array}{l}\mathbf{T g} \\
\left({ }^{\circ} \mathbf{C}\right)\end{array}$ & Reference \\
\hline Polyimide & 24.9 & 5.4 & 351.2 & 242 & (1) \\
\hline Aromatic polyurethane acrylates & 33.2 & - & 264.7 & - & (2) \\
\hline $\begin{array}{l}\text { Hyperbranched polyurethane } \\
\text { acrylate }\end{array}$ & 9.6 & 8.4 & 225.6 & - & (3) \\
\hline Epoxy-based resin & 55.0 & 12.1 & - & 111.6 & (4) \\
\hline $\begin{array}{c}\text { Acrylate-based photosensitive } \\
\text { resin }\end{array}$ & 44.9 & 5.5 & - & 83.3 & (5) \\
\hline pGE & 38.8 & 8.2 & 338 & 79.7 & (6) \\
\hline BMPR & 43.7 & 10 & - & 96.9 & (7) \\
\hline $3 \mathrm{DPT}$ & 22.8 . & 67.6 & - & 60 & (8) \\
\hline AWSO & - & - & - & -10 & (9) \\
\hline soy & - & - & - & 20 & $(10)$ \\
\hline SLR & 39.5 & 2.2 & 308.4 & 76.8 & (11) \\
\hline 3DPRTs & - & - & - & 55 & (12) \\
\hline AA-BPA-SMP & 11.4 & - & - & 71 & (13) \\
\hline BMA-PEGDMA & 29 & 40 & - & 60 & (14) \\
\hline HDDA & - & - & 230 & $48.7-76.3$ & (15) \\
\hline BHMP & 45.2 & - & 215 & 183 & (16) \\
\hline MCPP-3 & 38.5 & 5.9 & 313.0 & 80.0 & This work \\
\hline
\end{tabular}




\section{References}

(1) Guo, Y.; Ji, Z.; Zhang, Y.; Wang, X.; Zhou, F. Solvent-free and photocurable polyimide inks for 3D printing. J Mater Chem A 2017, 5, 16307-16314, DOI: 10.1039/c7ta01952a.

(2)Cheng, Q.; Zheng, Y.; Wang, T.; Sun, D.; Lin, R. Yellow resistant photosensitive resin for digital light processing 3D printing. $J$ Appl. Polym. Sci. 2020, 137, 48369, DOI:10.1002/app.48369.

(3)Xiang, H.; Wang, X.; Lin, G.; Xi, L.; Yang, Y.; Lei, D.; Dong, H.; Su, J.; Cui, Y.; Liu, X. Preparation, Characterization and Application of UV-Curable Flexible Hyperbranched Polyurethane Acrylate. Polymers 2017, 9, 552, DOI:10.3390/polym9110552.

(4)Li, Y.; Zheng, L.; Peng, S.; Miao, J.; Zhong, J.; Wu, L.; Weng, Z. Structure-Property Relationship of Stereolithography Resins Containing Polysiloxane Core-Shell Nanoparticles. ACS Appl. Mater. Inter. 2020, 12, 4917-4926, DOI:10.1021/acsami.9b20417.

(5)Zhang, X.; Xu, Y.; Li, L.; Yan, B.; Bao, J.; Zhang, A. Acrylate-based photosensitive resin for stereolithographic three-dimensional printing. J. Appl. Polym. Sci. 2019, 136, 47487, DOI:10.1002/app.47487.

(6) Ding, R.; Du, Y.; Goncalves, R. B.; Francis, L. F.; Reineke, T. M. Sustainable near UV-Curable Acrylates Based on Natural Phenolics for Stereolithography 3D Printing. Polym. Chem. 2019, 10 (9),1067-1077, DOI:10.1039/C8PY01652F.

(7) Guit, J.; Tavares, M. B. L.; Hul, J.; Ye, C.; Loos, K.; Jager, J.; Folkersma, R.; Voet, V. S. D. Photopolymer Resins with Biobased Methacrylates Based on Soybean Oil for Stereolithography. ACS Appl. Polym. Mater. 2020, 2 (2), 949-957, DOI:10.1021/acsapm.9b01143.

(8) Lu, C.; Wang, C.; Yu, J.; Wang, J.; Chu, F. Two-Step 3D-Printing Approach toward Sustainable, Repairable, Fluorescent Shape-Memory Thermosets Derived from Cellulose and Rosin. ChemSusChem 2020, 13 (5), 893-902, DOI:10.1002/cssc.201902191 
(9) Wu, B.; Sufi, A.; Ghosh Biswas, R.; Hisatsune, A.; Moxley-Paquette, V.; Ning, P.; Soong, R.; Dicks,A. P.; Simpson, A. J. Direct Conversion of Mcdonald's Waste Cooking Oil into a Biodegradable High-Resolution 3D-Printing Resin. ACS Sustainable Chem. Eng. 2020, 8 (2), 1171-1177, DOI:10.1021/acssuschemeng.9b06281.

(10) Miao, S.; Zhu, W.; Castro, N. J.; Nowicki, M.; Zhou, X.; Cui, H.; Fisher, J. P.; Zhang, L. G. 4D Printing Smart Biomedical Scaffolds with Novel Soybean Oil Epoxidized Acrylate. Sci. Rep. 2016, 6,27226, DOI: 10.1038/srep27226.

(11) Li, Y.; Zhong, J.; Wu, L.; Weng, Z.; Zheng, L.; Peng, S.; Zhang, X. High Performance Poss Filled Nanocomposites Prepared via UV-Curing Based on 3D Stereolithography Printing. Compos. Part A-Appl. S. 2019, 117, 276-286, DOI: 10.1016/j.compositesa.2018.11.024.

(12) Zhang, B.; Kowsari, K.; Serjouei, A.; Dunn, M. L.; Ge, Q. Reprocessable Thermosets for Sustainable Three-Dimensional Printing. Nat. Commun. 2018, 9 (1), 1831, DOI: $10.1038 / \mathrm{s} 41467-018-04292-8$.

(13) Yang, C.; Boorugu, M.; Dopp, A.; Ren, J.; Martin, R.; Han, D.; Choi, W.; Lee, H. 4D Printing Reconfigurable, Deployable and Mechanically Tunable Metamaterials. Mater. Horiz. 2019, 6 (6), 1244-1250, DOI: 10.1039/C9MH00302A.

(14) Zhang, B.; Zhang, W.; Zhang, Z.; Zhang, Y.-F.; Hingorani, H.; Liu, Z.; Liu, J.; Ge, Q. Self-Healing Four-Dimensional Printing with an Ultraviolet Curable Double-Network Shape Memory Polymer System. ACS Appl. Mater. Interfaces 2019, 11 (10), 10328-10336, DOI: 10.1021/acsami.9b00359.

(15) Wu, H.; Chen, P.; Yan, C.; Cai, C.; Shi, Y. Four-Dimensional Printing of a Novel Acrylate-Based Shape Memory Polymer Using Digital Light Processing. Mater. Design. 2019, 
171, 107704, DOI: 10.1016/j.matdes.2019.107704.

(16) Miao,J.; Peng,S.; Ge,M.; Li, Y.; Zhong,J.; Weng,Z.; Wu,L.; Zheng,L.Three-Dimensional

Printing Fully Biobased Heat-Resistant Photoactive Acrylates from Aliphatic Biomass, ACS

Sustainable Chem. Eng. 2020, 8 (2), 9415-9424, DOI: 10.1021/acssuschemeng.0c02168. 